\title{
Incidência natural e biologia de Trichogramma atopovirilia Oatman \& Platner, 1983 (Hymenoptera, Trichogrammatidae) em ovos de Anticarsia gemmatalis Hübner, 1818 (Lepidoptera, Noctuidae)
}

\author{
Carolina L. Cañete ${ }^{1,2}$ \\ Luís A. Foerster ${ }^{1,3}$
}

\begin{abstract}
Natural incidence and biology of Trichogramma atopovirilia Oatman \& Platner, 1983 (Hymenoptera, Trichogrammatidae) in eggs of Anticarsia gemmatalis Hübner, 1818 (Lepidoptera, Noctuidae). Trichogramma atopovirilia Oatman \& Platner is an egg parasitoid of the corn earworm Helicoverpa zea (Boddie) (Lepidoptera, Noctuidae), and has recently been collected from eggs of Anticarsia gemmatalis Hübner on soybeans. In order to evaluate the suitability of $A$. gemmatalis eggs as hosts of T. atopovirilia, field surveys were conducted in 1999 and 2000 on corn and soybeans, and a colony of the parasitoid was established in laboratory. At $25{ }^{\circ} \mathrm{C}$, development from oviposition to emergence lasted nine days and a sex-ratio of 0.58 (females:males) was obtained. Females lived significantly longer (11.4 days) when kept in ovipositional activity, than in the absence of host eggs (6.6 days). Total fecundity averaged 104.5 parasitized eggs, resulting in the emergence of 138.3 descendents. Mean daily fecundity was highest (30 eggs/female) on the first day. Oviposition continued until one day before the death of the females, however $70 \%$ of the eggs were laid during the first four days after emergence. A female-biased progeny was produced during the first three days of oviposition, whereas further ovipositions were male-biased. Females lived significantly longer when exposed to host eggs in comparison to females deprived of eggs. The results show that eggs of $A$. gemmatalis are suitable for the development of $T$. atopovirilia, and this parasitoid should be considered in future programs of biological control of the velvetbean caterpillar.
\end{abstract}

KEYwORDs. Biological control; egg parasitoid; soybeans; velvetbean caterpillar.

\section{INTRODUÇÃO}

A lagarta da soja Anticarsia gemmatalis Hübner, 1818 é o principal inseto desfolhador da cultura da soja no Brasil e pesquisas sobre a incidência de parasitismo em ovos dessa espécie são recentes (PolaszeK \& Foerster 1997; Foerster \& Avanci 1999). Dentre as espécies coletadas, Trichogramma atopovirilia Oatman \& Platner, 1983, uma espécie relatada anteriormente parasitando ovos de noctuídeos que atacam a cultura do milho (RESENDE \& CiOCIOLA 1996; ZuCCHI \& Monteiro 1997; PARON et al. 1998), foi encontrada pela primeira vez parasitando ovos de $A$. gemmatalis em soja no sul do Paraná (Foerster \& Avanci 1999). Milho e soja são comumente cultivados em conjunto no sul do Brasil, e a ocorrência de $T$. atopovirilia num hospedeiro não preferencial poderia ser um evento ocasional devido à proximidade das duas culturas.
Devido à inexistência de dados a respeito da viabilidade de ovos de A. gemmatalis como hospedeiros de T. atopovirilia, realizaram-se levantamentos de campo nas culturas do milho e da soja, com o objetivo de avaliar os níveis de parasitismo de $T$. atopovirilia em ovos de Helicoverpa zea (Boddie, 1850) e de A. gemmatalis. Para verificar se ovos de A. gemmatalis se constituem em um hospedeiro capaz de propiciar o desenvolvimento e a reprodução de T. atopovirilia, foi realizado um experimento de laboratório para determinar o tempo de desenvolvimento, a fecundidade e a longevidade desse parasitóide em ovos daquele hospedeiro.

\section{MATERIALE MÉTODOS}

Os experimentos de campo foram realizados na Fazenda Gralha Azul, localizada no Município de Fazenda Rio Grande a

\footnotetext{
1. Departamento de Zoologia, Universidade Federal do Paraná. Caixa Postal 19.020, 81531-990 Curitiba-PR, Brasil.

2. Bolsista da CAPES.

3. Pesquisador do CNPq.
} 
Tabela I. Parâmetros reprodutivos de T. atopovirilia em ovos de A. gemmatalis (temperatura: $25^{\circ} \mathrm{C}$; fotofase: 14 horas).

\begin{tabular}{lll}
\hline \multicolumn{1}{c}{ Pârametro avaliado } & \multicolumn{1}{c}{ Média \pm E.P. } & \multicolumn{1}{c}{ I.V. $^{1}$} \\
\hline $\mathrm{N}^{\mathrm{o}}$ de ovos parasitados / fêmea (total) & $104,5 \pm 10,23$ & $(60-165)$ \\
$\mathrm{N}^{\mathrm{o}}$ de descendentes / fêmea (total) & $138,3 \pm 12,24$ & $(92-208)$ \\
$\mathrm{N}^{\mathrm{o}}$ de ovos parasitados / fêmea / dia & $9,2 \pm 0,69$ & $(0-39)$ \\
$\mathrm{N}^{\mathrm{o}}$ de descendentes / fêmea / dia & $12,1 \pm 0,79$ & $(9-16)$ \\
\hline
\end{tabular}

${ }^{1}$ I.V. = Intervalo de variação

$30 \mathrm{~km}$ de Curitiba. Semanalmente, entre janeiro e março de 1999 e 2000, foram realizadas coletas nos estilo-estigmas de espigas de milho, onde são depositados os ovos de $H$. zea, e em folhas de soja para a coleta de ovos de A. gemmatalis. No laboratório, os ovos foram mantidos individualmente $a 25^{\circ} \pm 1^{\circ} \mathrm{C}$ em cápsulas de gelatina para verificar a presença de parasitóides. Registrouse o número e o sexo dos parasitóides emergidos de cada ovo. A identificação das espécies de Trichogramma foi realizada com base na morfologia da genitália dos machos (NAGARKATTI \& Nagaraja 1971; OAtMan \& Platner 1983).

A partir dos adultos de T. atopovirilia, estabeleceu-se no Laboratório de Controle Integrado de Insetos do Departamento de Zoologia da Universidade Federal do Paraná, uma criação em ovos de $A$. gemmatalis. Os experimentos foram realizados em câmara climatizada a $25^{\circ} \pm 1{ }^{\circ} \mathrm{C}$ e fotofase de 14 horas. Vinte cartelas de cartolina de $0,5 \times 1,0 \mathrm{~cm}$ contendo 50 ovos de $A$. gemmatalis foram individualizadas em tubos de ensaio de $0,5 \mathrm{x}$ $10,0 \mathrm{~cm}$ e expostas a uma fêmea de $T$. atopovirilia por 24 horas. Após esse período, as fêmeas foram eliminadas e os tubos contendo os ovos foram observados até a emergência dos parasitóides para determinar o tempo de desenvolvimento, o número médio e a razão sexual dos parasitóides emergidos por ovo. Após a emergência, os adultos foram separados em um casal por tubo de ensaio com 20 repetições e alimentados com um filete de mel depositado diariamente na parede do tubo. Dez casais receberam diariamente 50 ovos de $A$. gemmatalis colados em uma cartela de cartolina, substituída a cada 24 horas até a morte das fêmeas. Os outros 10 casais foram mantidos de forma idêntica, porém permaneceram sem a presença de ovos hospedeiros. Determinou-se a fecundidade das fêmeas de $T$. atopovirilia e comparou-se a longevidade de machos e fêmeas em atividade reprodutiva com a longevidade de fêmeas que não receberam ovos.

Comparações estatísticas entre a longevidade de machos e fêmeas e entre fêmeas em atividade de parasitismo e na ausência de ovos hospedeiros foram feitas pelo teste "t" de Student.

Exemplares de T. atopovirilia montados em lâminas foram depositados na coleção de referência do Laboratório de Controle Integrado de Insetos do Departamento de Zoologia da Universidade Federal do Paraná e na coleção entomológica de Trichogramma da Escola Superior de Agronomia "Luiz de Queiroz" da Universidade de São Paulo.

\section{RESULTADOS E DISCUSSÃO}

Nos levantamentos de campo, adultos de $T$. atopovirilia foram obtidos tanto de ovos de $H$. zea coletados no milho, quanto de A. gemmatalis em soja. Em 1999, a incidência natural de parasitismo em ovos de $H$. zea foi de $5,6 \%$, com 41 ovos parasitados de um total de 734 ovos coletados. Dos ovos parasitados, $70,7 \%$ deram origem a $T$. pretiosum Riley, $1879 \mathrm{e}$ $29,3 \%$ a T. atopovirilia. Para a mesma época do ano (fevereiro), Tironi \& Ciociola (1994) registraram em Minas Gerais 7,3\% de parasitismo em ovos de $H$. zea, sendo que T. pretiosum foi a espécie predominante, com mais de $90 \%$ dos parasitóides emergidos. Em 2000, de 343 ovos coletados na cultura do milho, apenas três se encontravam parasitados, sendo dois por $T$. pretiosum e um por T. atopovirilia. Em soja, o índice de parasitismo natural em ovos de $A$. gemmatalis em 2000 foi de 4,8 \%, sendo T. atopovirilia a terceira espécie em abundância, após T. pretiosum e T. acacioi Brun, Moraes \& Soares, 1984 (dados não publicados).

Em laboratório a $25^{\circ} \mathrm{C}$, o tempo de desenvolvimento de $T$. atopovirilia em ovos de $A$. gemmatalis foi de nove dias para todas as repetições, não havendo na literatura resultados sobre esse parâmetro para $T$. atopovirilia. A razão sexual dos parasitóides emergidos foi de 0,58 (fêmeas:machos). Fêmeas

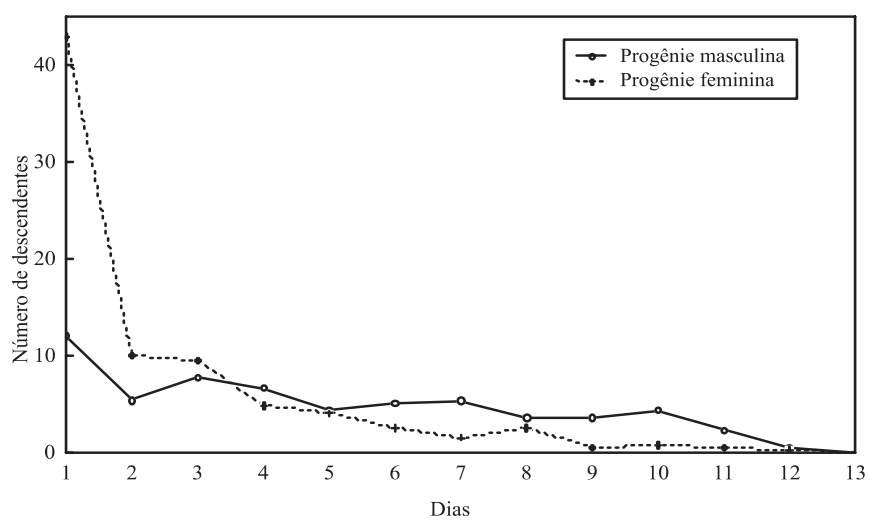

Fig. 1. Média de progênie masculina e feminina produzida durante a vida de $T$. atopovirilia em ovos de A. gemmatalis (Temperatura: $25^{\circ} \mathrm{C}$; Fotofase: 14 horas). 
de T. atopovirilia parasitaram em média 104,5 ovos de $A$. gemmatalis, resultando numa média de 138,3 descendentes por fêmea (Tabela I), ou seja, 1,3 parasitóides por ovo hospedeiro. Em média, cada fêmea parasitou 9,2 ovos de $A$. gemmatalis por dia, gerando 12,1 descendentes.

O número de ovos parasitados por fêmea foi mais que o dobro do encontrado por ReSENDE \& Ciociola (1996), os quais obtiveram uma média de 45,2 ovos de $H$. zea parasitados por fêmea de T. atopovirilia. A fecundidade registrada para essa espécie foi semelhante à observada por AvANCI (1999) para $T$. pretiosum, também em ovos de A. gemmatalis, a qual apresentou uma média de 128,5 ovos parasitados e 1,9 parasitóides por ovo. A diferença na capacidade de parasitismo de T. atopovirilia em relação àquela obtida por RESENDE \& Ciociola (1996) pode estar relacionada à procedência das linhagens do parasitóide, uma vez que linhagens de Trichogramma de diferentes localidades apresentam variações em sua biologia (BLEICHER \& PARRA 1989). A diferença encontrada entre os dados deste trabalho e os de RESENDE \& Ciociola (1996) pode também estar relacionada à espécie hospedeira, visto que estes autores utilizaram ovos de $\mathrm{H}$. zea como hospedeiro. Diferenças na biologia de T. pretiosum em função do hospedeiro foram também constatadas por KuHLMANn \& Mills (1999).

Nos três primeiros dias de oviposição, a progênie foi predominantemente do sexo feminino, especialmente no primeiro dia. A partir do quinto dia de oviposição, o número de ovos parasitados diminuiu e predominou a ocorrência de descendentes machos (Fig. 1).

Do total de ovos parasitados pelas fêmeas de $T$. atopovirilia, 29,5\% ocorreu no primeiro dia de oviposição e cerca de $70 \%$ dos ovos foram parasitados até o quarto dia de vida das fêmeas (Fig. 2), valor semelhante ao obtido por Resende \& Ciociola (1996) para essa espécie em ovos de $H$. $z e a$.

Nas primeiras 24 horas após a emergência, as fêmeas de $T$. atopovirilia parasitaram em média 30 ovos de $A$. gemmatalis (Fig. 2); esse valor é acentuadamente maior do que aquele registrado por PARON et al. (1998), que obtiveram para fêmeas de mesma idade e durante o mesmo tempo de exposição, uma média de apenas 8,0 ovos parasitados. Tal diferença provavelmente se deve ao fato das fêmeas de T. atopovirilia

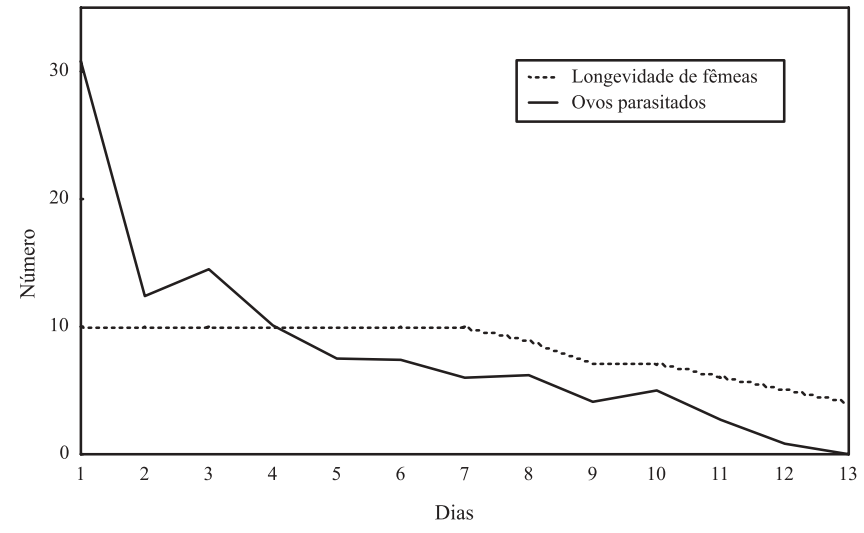

Fig. 2. Média de ovos parasitados e longevidade de T. atopovirilia em ovos de $A$. gemmatalis (Temperatura: $25^{\circ} \mathrm{C}$; Fotofase: 14 horas).

utilizadas nesse estudo terem sido obtidas de ovos de Anagasta kuehniella (Zeller, 1879) (Lepidoptera, Pyralidae), um hospedeiro de tamanho reduzido em comparação aos ovos de noctuídeos, resultando em fêmeas de menor tamanho e, por conseguinte, com menor capacidade de parasitismo. Essa hipótese é corroborada pelos resultados de Marston \& ERTLE (1973) que obtiveram 2,4 vezes mais ovos parasitados de Trichoplusia ni (Hübner, 1803) quando as fêmeas de T. minutum Riley, 1879 foram obtidas de ovos desse hospedeiro, do que quando criadas em ovos de Sitotroga cerealella (Olivier, 1789) (Lepidoptera, Gelechiidae). Os padrões de oviposição, assim como da razão sexual são importantes em programas de controle biológico, nos quais se buscam alta fecundidade e predominância de exemplares do sexo feminino para liberações no campo.

Apesar da redução na fecundidade de T. atopovirilia a partir do quinto dia, as fêmeas permaneceram reprodutivamente ativas até onze dias após a emergência (Fig. 2). Esse fato é relevante para o uso desses parasitóides no controle biológico de pragas agrícolas, uma vez que o período de atividade reprodutiva é fundamental para a eficiência da espécie como agente de controle.

A longevidade das fêmeas de T. atopovirilia foi significativamente maior (11,4 dias) quando receberam diariamente ovos de $A$. gemmatalis durante o estágio adulto,

Tabela II. Longevidade (em dias) de fêmeas de T. atopovirilia na presença e ausência de hospedeiros e de machos na presença de hospedeiros (temperatura: $25^{\circ} \mathrm{C}$; fotofase: 14 horas).

\begin{tabular}{lll}
\hline \multicolumn{1}{c}{ Longevidade } & Média \pm E.P. ${ }^{1}$ & $(\text { I.V. })^{2}$ \\
\hline Fêmeas com hospedeiro & $11,4 \pm 0,64 \mathrm{a}$ & $(8-13)$ \\
Fêmeas sem hospedeiro & $6,6 \pm 0,70 \mathrm{~b}$ & $(2-12)$ \\
\hline Machos acasalados & $7,7 \pm 0,60$ & $(4-10)$ \\
\hline
\end{tabular}

${ }^{1}$ Médias seguidas de mesma letra minúscula na coluna não diferem entre si pelo teste de "t" a $5 \%$ de probabilidade.

${ }^{2}$ I.V. = Intervalo de variação 
em comparação com fêmeas que não receberam ovos (6,6 dias) (Tabela 2). PAK \& OATMAN (1982) descreveram que fêmeas de $T$. pretiosum e T. brevicapillum Pinto \& Platner, 1978 se alimentam do líquido que extravasa dos ovos por ocasião da inserção do ovipositor. Esse fluído pode se constituir em um suplemento alimentar que prolonga a sobrevivência das fêmeas. Da mesma forma, fêmeas de T. pretiosum e T. rojasi Nagaraja \& Nagarkatti, 1973 também sobreviveram por mais tempo quando na presença de ovos hospedeiros do que fêmeas que não receberam ovos (Avanci 1999). Por outro lado, no parasitóide de ovos Gryon obesum Masner, 1983 (Hymenoptera, Scelionidae) (EHLER et al. 2002) e no parasitóide larval Glyptapanteles muesebecki (Blanchard, 1947) (Hymenoptera, Braconidae) (FoERSTER et. al. 1999), a longevidade das fêmeas em atividade de parasitismo foi significativamente reduzida em relação àquelas reprodutivamente inativas.

Os resultados obtidos quanto ao tempo de desenvolvimento, fecundidade e longevidade de T. atopovirilia demonstram que esse parasitóide apresenta potencial para ser utilizado no controle biológico da lagarta da soja, não diferindo dos parâmetros biológicos de T. pretiosum (Avanci 1999), espécie mais abundante de Trichogramma em ovos de $A$. gemmatalis (FoERSTER \& AvANCI 1999).

Agradecimentos. Este projeto de pesquisa foi financiado pelo $\mathrm{CNPq}$ e Fundação Araucária, a quem os autores expressam o seu agradecimento, e à Marion do Rocio F. Avanci pelo auxílio nas coletas de campo e na realização dos experimentos de laboratório.

\section{REFERÊNCIAS}

Avanci, M. R. F. 1999. Incidência de parasitóides em ovos de Anticarsia gemmatalis Hübner, 1818 (Lepidoptera: Noctuidae); biologia e competitividade entre duas espécies de Trichogramma (Hymenoptera: Trichogrammatidae). Dissertação de Mestrado. Universidade Federal do Paraná, Curitiba, 86 p.

Bleicher, E. \& J. R. P. PARRA. 1989. Espécies de Trichogramma parasitóides de Alabama argillacea. I. Biologia de três populações. Pesquisa Agropecuária Brasileira 24: 929-940.

Ehler, L. E.; L. B. Maller \& Y. Hirose. 2002. Reproductive biology of
Gryon obesum Masner (Hymenoptera: Scelionidae). Journal of Hymenoptera Research 11: 1-4.

Foerster, L. A. \& M. R. F. AvanCI. 1999. Egg parasitoids of Anticarsia gemmatalis Hübner (Lepidoptera: Noctuidae) in soybeans. Anais da Sociedade Entomológica do Brasil 28: 545-548.

Foerster, L. A.; A. K. Doetzer \& M. R. F. Avanci. 1999. Capacidade reprodutiva e longevidade de Glyptapanteles muesebecki (Blanchard) (Hymenoptera: Braconidae) parasitando lagartas de Pseudaletia sequax Franclemont (Lepidoptera: Noctuidae). Anais da Sociedade Entomológica do Brasil 28: 485-490.

Kuhlmann, U. \& N. J. Mills. 1999. Comparative analysis of the reproductive attributes of three comercially-produced Trichogramma species (Hymenoptera: Trichogrammatidae). Biocontrol Science and Technology 9: 335-346.

Marston, N. \& L. R. Ertle. 1973. Host influence on the bionomics of Trichogramma minutum. Annals of the Entomological Society of America 66: 1155-1162.

Nagarkati, S. \& H. Nagaraja. 1971. Redescriptions of some known species of Trichogramma (Hym., Trichogrammatidae), showing the importance of the male genitalia as a diagnostic character. Bulletin of Entomological Research 61: 13-31.

PAK, G. A. \& E. R. OATMAn. 1982. Comparative life table, behavior and competition studies of Trichogramma brevicapillum and T. pretiosum. Entomologia experimentalis et applicata 32: 68-79.

Oatman, E. R. \& G. R. Platner. 1983. A new species of Trichogramma (Hymenoptera: Trichogrammatidae), with notes on other species collected in Guatemala. Proceedings of the Entomological Society of Washington 85: 710-713.

Paron, M. F. O.; I. Cruz \& A. I. Ciociola. 1998. Efeito de genótipos de milho no parasitismo por Trichogramma spp. em ovos de Helicoverpa zea (Boddie). Anais da Sociedade Entomológica do Brasil 27: 435-441.

Polaszer, A. \& L. A. Foerster. 1997. Telenomus cyamophylax, n. sp. (Hymenoptera: Scelionidae) attacking eggs of the velvetbean caterpillar, Anticarsia gemmatalis Hübner (Lepidoptera: Noctuidae). Anais da Sociedade Entomológica do Brasil 26: 177-181.

Resende, D. L. M. C. \& A. I. Ciociola. 1996. Capacidade de parasitismo de Trichogramma atopovirilia Oatman \& Platner, 1983 (Hymenoptera: Trichogrammatidae) em ovos de Helicoverpa zea (Boddie, 1850) (Lepidoptera: Noctuidae) em diferentes temperaturas. Ciência e Agrotecnologia 20: 421-424.

Tironi, P. \& A. I. Ciociola. 1994. Parasitismo natural de ovos de Helicoverpa zea (Boddie, 1850) (Lepidoptera: Noctuidae) por duas espécies de Trichogramma (Hymenoptera: Trichogrammatidae) em culturas de milho em Lavras, MG. Ciência e Prática 18: 61-67.

Zucchi, R. A. \& R. C. Monteiro. 1997. O gênero Trichogramma na América do Sul, p. 41-66. In: J. R. P. PARRA \& R. A. ZuCCHI (eds.). Trichogramma e o Controle Biológico Aplicado. Piracicaba, FEALQ, 324 p. 